\title{
Identification of proteins associated with pediatric bilateral Wilms tumor
}

\author{
ZECHEN YAN ${ }^{1}$, QINGJUN MENG ${ }^{1}$, JINJIAN YANG ${ }^{1}$, JUNJIE ZHANG ${ }^{1}$, WEI ZHAO ${ }^{1}$, \\ FEI GUO ${ }^{1}$, DONGJIAN SONG ${ }^{1}$, YUXIAO ZHAN ${ }^{1}$, DANDAN FAN ${ }^{2}$, RUIYI ZHOU ${ }^{1}$, \\ SHUQIANG ZUO $^{1}$, ZEHUA WANG ${ }^{1}$, JIEKAI YU ${ }^{3}$, SHU ZHENG $^{3}$ and JIAXIANG WANG ${ }^{1}$ \\ ${ }^{1}$ Department of Surgery, The First Affiliated Hospital of Zhengzhou University; ${ }^{2}$ Henan Academy \\ of Medical and Parmaceutical Science, Zhengzhou University, Zhengzhou, Henan 450052; ${ }^{3}$ Institute of Cancer, \\ The Second Affiliated Hospital, College of Medicine, Zhejiang University, Hangzhou, Zhejiang 310000, P.R. China
}

Received April 19, 2016; Accepted September 15, 2016

DOI: $10.3892 / \mathrm{ol} .2016 .5306$

\begin{abstract}
Wilms tumor (WT) is the most common cancer that primarily develops in abdominal solid organ of children. It has no incipient symptom, and the most frequent symptoms are a painless, palpable abdominal mass. Proteomics technology was used to select the differentially expressed proteins of bilateral Wilms tumor (BWT). Ten serum samples of children with BWT were chosen, 20 serum samples of children with unilateral WT (UWT) and 20 serum samples of healthy children were selected, and proteomics technology was used to detect and collect data. Using bioinformatics, the data were analyzed and 10 difference peaks were obtained $(\mathrm{P}<0.01)$. Non-linear support vector machine was used to classify and to select the composite pattern with the highest Youden's index, and one differentially expressed protein with $\mathrm{m} / \mathrm{z}$ of $5,648 \mathrm{kDa}$ was obtained. A significantly high expression in children with BWT was obtained, and the expression intensity was also significantly $(3,889.36 \pm 1,796.83)$ higher for children with BWT compared to those with UWT $(2,886.81 \pm 1,404.65)$ and healthy children $(432.21 \pm 730.42)$. Matrix-assisted laser desorption ionization/time-of-flight ionization/time-of-flight mass spectrometry was used for identification of the peak, and the peak was further identified as apolipoprotein C-III (APO C-III) by western blot analysis. In conclusion, to the best of our knowledge, a differentially expressed protein of APO C-III of BWT was obtained through proteomics technology for the first time, and it is expected to be a new marker for the early diagnosis and prognosis of BWT.
\end{abstract}

Correspondence to: Professor Jiaxiang Wang, Department of Surgery, The First Affiliated Hospital of Zhengzhou University, 1 Jianshe East Road, Zhengzhou, Henan 450052, P.R. China E-mail: jiaxiang_wang1@163.com

Key words: apolipoprotein C-III , proteomics, biological markers, nephroblastoma

\section{Introduction}

Wilms tumor (WT) is the most common cancer that primarily develops in abdominal solid organ of children, with an incidence rate of 2.5/1 million in China $(1,2)$. It has no incipient symptom, and the most frequent symptoms are a painless, palpable abdominal mass. Bilateral WT (BWT) accounts for $5-7 \%$ and is more complex than unilateral WT (UWT) in diagnosis and treatment $(1,2)$. However, at present no tumor marker has been identified to diagnose BWT accurately. With the continuous development of proteomics technologies in recent years, new ideas and methods for detecting differential expression protein of WB especially BWT have been adopted $(3,4)$.

From January, 2009 to December, 2013, we collected serum samples of BWT, UWT and healthy children, and screened BWT differential expression protein with surface-enhanced laser desorption/ionisation time-of-flight mass spectrometry (SELDI-TOF MS), to determine the differential expression protein associated with BWT.

\section{Materials and methods}

Serum analysis. Serum samples were selected from 10 cases of children with BWT (BWT group), 20 cases of children with UWT (UWT group) and 20 cases of healthy children (control group). All of the children were sampled after overnight fasting. The 3 males and 2 females in the BWT group, aged 7-48 months (average 34.20 \pm 12.41 months), were confirmed as BWT pathologically. Six males and 4 females in the UWT group, aged 4-56 months (average 41.20 \pm 10.12 months), were confirmed as UWT pathologically. The 5 males and 5 females in the control group were aged 4-74 months (average of 34.53 \pm 15.52 months). According to WT staging criteria, BWT was in stage V and UWT in stages I-IV. The serum samples standing at $25^{\circ} \mathrm{C}$ for 1-2 $\mathrm{h}$ were centrifuged for $20 \mathrm{~min}$ at 2,500 x g, and the supernatant was placed in Eppendorf tubes and preserved at $-80^{\circ} \mathrm{C}$.

The present study was approved by the Ethics Committee of Zhengzhou University (Zhejiang, China). Written informed consent was obtained from the patient's relatives and/or guardians. 
Reagents and instruments. Urea, NaNC, dithiothreitol, acetonitrile, 3-[(3-cholamidopropyl) dimethylammonio)1-propanesulfonate, trifluoroacetic acid, sinapinic acid and trypsin were purchased from Sigma (St. Louis, MO, USA). Spectra Multicolor Low Range Protein Ladder and SPD SpeedVac were purchased from Thermo Fisher Scientific (Waltham, MA, USA). PBSII SELDI-TOF MS and the WCX2 protein chip were purchased from Ciphergen Biosystems Inc. (Fremont, CA, USA). Ammonium persulfate, 2X/4X Laemmli Sample Buffer, 10X Tris/Glycine/SDS, TEMED, Powerpac Universal and Mini-PROTEAN Tetra vertical electrophoresis system were purchased from Bio-Rad (Berkeley, CA, USA). Matrix-assisted laser desorption ionization/time-of-flight mass spectrometry (MALDI-TOF MS) was purchased from Kratos Analytica Inc. (Spring Valley, NY, USA). Uhraflex III MALDI-TOF/TOF MS were purchased from Bruker Corp. (Bremen, Germany).

Screening serum differential protein markers with SELDI-TOF MS. Sera taken from the three groups were thawed on ice-bath for 30-60 min and centrifuged at 8,000 x g. A total of $5 \mu \mathrm{l}$ of serum was taken and added to $10 \mu \mathrm{l}$ U9 serum conditioning fluid, mixed fully at 600 times/min for total of $30 \mathrm{~min}$ on shaker at $4^{\circ} \mathrm{C}$. The serum conditioned with U9 was diluted with binding buffer to $200 \mu \mathrm{l}$, and incubated on a shaker at $4^{\circ} \mathrm{C} 600$ times/min for $2 \mathrm{~min}$ and WCX2 protein chip was applied (1). All-in-One standard protein chip with known molecular weight was used to correct the error of SELDI MS system to $<0.1 \%$, and then the protein chip well connected with protein was put into the mass spectrometer for detection $(2,3)$. Ciphergen Biosystems Inc. (Version 3.1) was used to correct the data collected, to homogenize the total ionic strength and molecular weight, and Biomarker wizard and ZUCI protein chip data analysis system were used for de-noising of the signal of original data with non-sampling discrete wavelet transform approach. A mark of signal strength was adjusted according to the three peak values marked and appeared in all selected spectra. The baseline of spectrum was corrected based on the monotonous and aligned minimum curve and quality, and peaks with signal-to-noise ratio $>2$ were filtered. In the cluster analysis, the minimum threshold was set to $10 \%$, and peaks with proton number/charge number $(\mathrm{m} / \mathrm{z})$ difference $<0.3 \%$ in each sample were clustered as a category (4).

Separation of the target protein with SDS-PAGE. Gene electrophoresis was performed using a protein marker (1.7-40 kDa) in the first lane, and serum samples of the tumor and control groups were added in other lanes ( $5 \mu \mathrm{l}$ in each lane) at $90 \mathrm{~V}$. The spacer gel was removed and dyed using Coomassie blue coloring agent overnight, and then de-colored for $1.5 \mathrm{~h}$. The de-coloring liquid was discarded to continue decolorizing for $2.5 \mathrm{~h}$, by comparing with a protein ladder. The corresponding target protein bands were incised in aseptic condition, chopped and preserved for protein identification (5).

Determination of candidate protein markers by MALDITOF MS. The purified protein fluid at different periods of time were, respectively, pulled into an integrated centrifuging enrichment system (SPD SpeedVac) and freeze-dried to $20 \mu \mathrm{l}$. Freeze-dried proteins $(1.5 \mu \mathrm{l})$ were mixed with $1.5 \mu \mathrm{l}$
Table I. Expression of m/z in 5,648 kDa peptide fragment and protein in BWT group and control group (mean \pm SD).

\begin{tabular}{lc}
\hline Group & Expression intensity \\
\hline BWT & $3,889.36 \pm 1,796.83^{\mathrm{a}}$ \\
Normal control & $432.21 \pm 730.42$ \\
\hline
\end{tabular}

Compared to the normal control group, ${ }^{a} \mathrm{P}<0.01$. SD, standard deviation; BWT, bilateral Wilms tumor.

Table II. Expression of $\mathrm{m} / \mathrm{z}$ in 5,648 $\mathrm{kDa}$ peptide fragment and protein in the BWT and UWT groups (mean \pm SD).

\begin{tabular}{lc}
\hline Group & Expression intensity \\
\hline BWT & $3,889.36 \pm 1,796.83^{\mathrm{a}}$ \\
UWT & $2,886.81 \pm 1,404.65$
\end{tabular}

Compared to the UWT group, ${ }^{\mathrm{a}} \mathrm{P}<0.01$. $\mathrm{SD}$, standard deviation; BWT, bilateral Wilms tumor; UWT, unilateral WT.

Table III. Expression of $\mathrm{m} / \mathrm{z}$ in 5,648 $\mathrm{kDa}$ peptide fragment and protein in the UWT and control groups (mean $\pm \mathrm{SD}$ ).

\begin{tabular}{lc}
\hline Group & Expression intensity \\
\hline UWT & $2,886.81 \pm 1,404.65^{\mathrm{a}}$ \\
Normal control & $432.21 \pm 730.42$ \\
\hline
\end{tabular}

Compared to the normal control group, ${ }^{\mathrm{a}} \mathrm{P}<0.01$. UWT, unilateral Wilms tumor.

CHCA, respectively, and cytochrome $\mathrm{C}$ (molecular weight of 12,361.96) + CHCA and insulin (molecular weight of $5,734.51)+$ CHCA were used to correct the samples. The target board was placed in MALDI-TOF MS for detection and peaks of the target protein were tracked again.

Enzymolysis of the target proteins. The protein containing the target protein was added with ultrapure water $(40 \mu \mathrm{l})$ and $4 \mu 10.1$ mol DTT solution of certain concentration was placed in $37^{\circ} \mathrm{C}$ warm water for $1 \mathrm{~h}$. The $1.6 \mu \mathrm{l} \mathrm{IAM}$ was added into the mixture and placed in the dark for $1 \mathrm{~h}$, and $1.6 \mu \mathrm{l}$ of $1 \mathrm{~mol}$ DTT solution, $150 \mu \mathrm{l}$ of $0.1 \mathrm{~mol} \mathrm{NH}_{4} \mathrm{HCO}_{3}$ (ammonium bicarbonate) and $2 \mu \mathrm{l}$ trypsin were added successively, and placed in $37^{\circ} \mathrm{C}$ warm water overnight (6-8 h).

Identification of the target protein. Trypsin samples were digested and the substrate CCA was mixed and applied to the target sample for natural drying. The protein chip was placed into MALDI-TOF/TOF MS to detect the peptide fragment of enzymolysis, and Mascot search software (Matrix Science, Ltd., London, UK) was used to retrieve the proteins that may match with the identified peptides and amino acids in the SwissProt database (6). 
Table IV. Protein or peptide segments with $\mathrm{m} / \mathrm{z}$ values of 5,648 $\mathrm{kDa}$ in the target protein.

\begin{tabular}{lcccr}
\hline $\mathrm{m} / \mathrm{z}, \mathrm{kDa}$ & Protein name & Sequence identified & Sequence coverage (\%) & Score \\
\hline 5,648 & Apolipoprotein C-III & TAKDALSSVQESQVAQQARGWVTDG & $57.22 \%$ & 52.11 \\
& & FSSLKDYWSTVKDKFSEFWDLDPE & & \\
\hline
\end{tabular}

Confirmation of candidate protein biomarkers by western blotting. First, SDS-PAGE was used to extract and separate total protein from the serum sample. Antigen-antibody immunological reaction was carried out after semi-dry trarsmembrane, GAPDH was used as internal reference. The chemiluminescence, developing and photographic fixing operation were carried out, and negative film was retained. After scanning negative film, Image pro plus 6.0 software (Media Cybernetics, Silver Spring, MD, USA) was used to measure the gray value of each negative protein band of the film, and the gray values were divided into the BWT, UWT and healthy control groups, and the gray values were statistically assessed.

Statistical analysis. After noise filtering and clustering analysis, the Wilcoxon rank sum test was conducted against the $\mathrm{m} / \mathrm{z}$ peaks selected, and the t-test was conducted against the data of each group. $\mathrm{P}<0.01$ was considered statistically significant.

\section{Results}

Screening serum differential protein markers with SELDI-TOF MS. SELDI-TOF MS was applied to screen the serum samples of each group, to obtain a series of data of protein expression intensity, and then the Wilcoxon rank sum test $(\alpha=0.01)$ was conducted against data of the BWT, UWT and healthy control groups, to screen the $\mathrm{m} / \mathrm{z}$ peaks of differential expression protein. Fourteen differential protein peaks were selected from the BWT and healthy control groups, 8 of which were of high level expression in the BWT group and 4 were high level expression in the healthy control group. Ten differential protein peaks were selected from the BWT and UWT groups, 8 of which were of high level expression in the BWT group and 2 of high level expression in the UWT group. A total of 13 differential protein peaks were selected from the UWT and healthy control group, 11 of which were of high level expression in the BWT group and 2 of high level expression in the UWT group. By comparing the differential protein peaks of the above groups, the difference of the $5,648 \mathrm{kDa} \mathrm{m} / \mathrm{z}$ differential expression proteins selected between the BWT group and healthy control group was of statistical significance $(\mathrm{P}<0.01)$ (Table I), and the difference between the BWT and UWT groups was of statistical significance $(\mathrm{P}<0.01)$ (Table II), and the difference between UWT group and the normal group was of statistical significance $(\mathrm{P}<0.01)($ Table III). The protein expression in the BWT, UWT and healthy control group were 100,200 and 300, respectively.

Identification of the target proteins. Peptide fragments of $\mathrm{m} / \mathrm{z}$ of the $5,648 \mathrm{kDa}$ or protein samples were digested by enzymolysis, respectively, and peptide fragments were detected

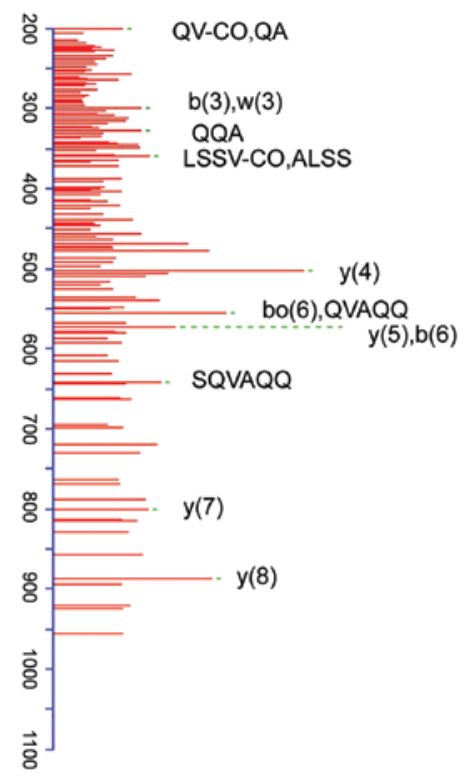

Figure 1. MS spectra of the target protein from the 5,648 kDa protein. MS, mass spectrometry.

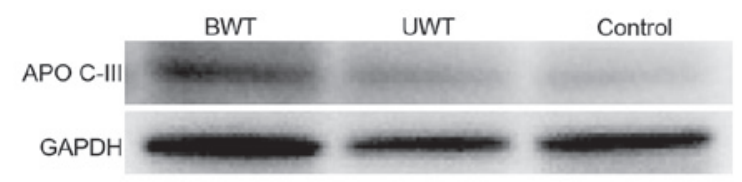

Figure 2. Representative western blot images of apolipoprotein C-III (APO C-III) and reference bands of bilateral Wilms tumor (BWT), unilateral WT (UWT) and control groups.

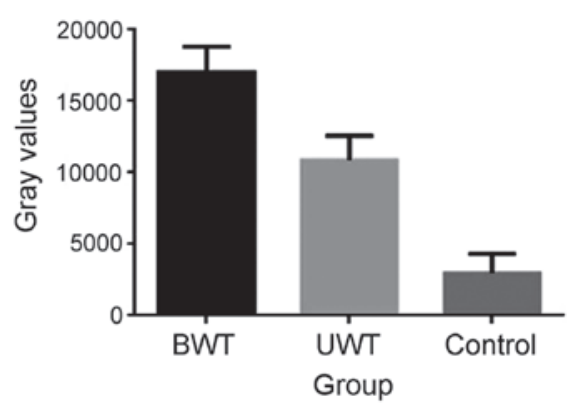

Figure 3. Quantitative analysis of the gray values of the western blot bands of apolipoprotein C-III in bilateral Wilms tumor (BWT), unilateral WT (UWT) and control groups.

with the 2D-LC-LTQ-MS system (Fig. 1). According to the results of importing peptide fragments to SEQUEST retrieval program and retrieving in Bioworks database, the peptides 
were apolipoprotein C-III (APO C-III) with a coverage rate of $57.22 \%$ (Table IV).

Confirmation of candidate protein biomarkers. A total of 18 patients were randomly selected to carry out western blot analysis, including 6 samples from the BWT group, 6 samples from the UWT group, and 6 samples from the control group. Target protein APO C-III appeared in all the bands, statistical analysis on the band gray value of target protein in the 3 groups of sera. The result indicated that the differences between the APO C-III gray values of the APO and control groups, and the differences between the APO C-III gray values of the BWT and UWT groups have statistical significance (Figs. 2 and 3).

\section{Discussion}

WT is the most common abdominal solid cancer of children, mostly discovered by parents during bathing or while dressing the children (7). BWT is rare, accounting for $\sim 5 \%$ of all patients with WT, and is more complex in its diagnosis and treatment compared with UWT (7). In addition, according to WT staging criteria formulated by the Children Tumor Institute based on surgery and histopathology, BWT was confirmed as stage V and was worse than stages I-IV of WT in prognosis (8). There is currently no report available on the significant improvement of the prognosis by early WBT diagnosis and treatment or WBT tumor marker. Therefore, to improve the early diagnostic rate and improve the prognostic effect of children with BWT, detecting the differential protein expression of BWT is particularly important (8).

Proteomics refers to all the proteins and existing ways of genome expression. SELDI-TOF MS technology can detect the molecular weight and expression level of protein of all types in the samples under test, and MALDI-TOF/TOF MS technology can detect the protein molecular weight, structure and other information of the samples under test. These technologies have wide prospect of application in detecting tumor markers, and with the emergence and development of the technologies, and new ideas and methods of detecting differential expression proteins of WB especially of BWT were developed (9).

Protein of $\mathrm{m} / \mathrm{z}$ of $5,648 \mathrm{kDa}$ obtained was APO C-III, and APO C-III with intensities of the protein expression of $(3,889.36 \pm 1,796.83),(2,886.81 \pm 1,404.65)$ and $(432.21 \pm 730.42)$, respectively, in BWT, UWT and the control groups. Pairwise comparison was conducted against the relative expression levels of the three groups of proteins stated above, and the difference was statistically significant $(\mathrm{P}<0.01)$. Currently, it is believed that histopathological features and staging are the most important factors determining prognosis effect of children with WT (10). According to WT staging criteria formulated by the Children Tumor Institute based on surgery and histopathology, BWT was in stage $\mathrm{V}$ and UWT in stages I-IV. In BWT group (stage V), intensity of protein expression of $\mathrm{m} / \mathrm{z}$ of $5,648 \mathrm{kDa}$ in the BWT group was significantly higher than that in the UWT group (stages I-IV), and intensity of protein expression of UWT and BWT groups was significantly higher than that in the healthy control group, indicating that the protein may be positively associated with WT development. According to previous studies, APO C-III has low level of expression in the serum of healthy children and a high level of expression in the serum of children in stages I-IV, and the intensity of protein expression increases gradually. Serum of the children in teh BWT group (stage V) was studied, showing that the intensity of APO C-III expression was significantly higher than that of the UWT group (stages I-IV), confirming that the protein is a type of WT protein marker and its protein expression intensity is positively correlated with WT staging (11). In addition, we verified the diverse expression of APO C-III in BWT, UWT and healthy control groups to show that APO C-III can be used as a serum protein marker of WT, especially BWT.

APO C-III is a type of protein in lipoprotein and can be divided into A, B, C, D, E and other major categories $(11,12)$. It acts as a lipid carrier in plasma and can identify APO C-III receptors and regulate plasma lipoprotein metabolism enzyme activity (12). APO C-III is a protein produced mainly by liver and can inhibit the combination between APO E and liver APO E receptor, thus affecting the intake of lipoprotein with very low density and chylomicron by the liver. Additionally, protein can inhibit the activity of hepatic lipase, thus inhibiting the conversion and metabolism of triglyceride lipoprotein remnants (13).

An increase in the level of APO C-III expression in cancer patients is likely to be caused by the inhibited activity of immune cells in the body and functional decline, resulting in excessive high concentrations of cholesterol and insulin in blood not excluding raised level of APO C-III expression due to stronger exuberance of metabolic activities of tumor tissues than other tissues, and it has no clear causality with BWT development (14). In addition, use of APO C-III expression in diagnosis initially requires identification of metabolic syndrome and the disease affecting homergy of the human body (15).

In conclusions, in the present study, a differential expression of APO C-III of $5,648 \mathrm{kDa} \mathrm{m} / \mathrm{z}$ of BWT was identified using proteomic technology as APO C-III. APO C-III has good value and prospect of application in the diagnosis and prognosis of WT, especially BWT, and is expected to become a new marker for early diagnosis and prognosis (16). However, because of the low incidence of BWT, relatively few samples were involved in the present study, and the unclear associations between APO C-III and BWT pathogenesis, and reliability of the differential expression APO C-III still remain to be studied further.

\section{Acknowledgements}

The present study was supported by the National Natural Science Foundation of China (no. 81071782), and the Medical Science and Technique Foundation of Henan Province (no. 201503055).

\section{References}

1. Wilde JC, Aronson DC, Sznajder B, Van Tinteren H, Powis M, Okoye B, Cecchetto G, Audry G, Fuchs J, Schweinitz DV, et al: Nephron sparing surgery (NSS) for unilateral Wilms tumor (UWT): The SIOP 2001 experience. Pediatr Blood Cancer 61: 2175-2179, 2014.

2. Venkatramani R, Malogolowkin MH and Mascarenhas L: Treatment of multiply relapsed Wilms tumor with vincristine, irinotecan, temozolomide and bevacizumab. Pediatr Blood Cancer 61: 756-759, 2014. 
3. Kamai T, Tomosugi N, Abe H, Kaji Y, Oyama T and Yoshida K Protein profiling of blood samples from patients with hereditary leiomyomatosis and renal cell cancer by surface-enhanced laser desorption/ionization time-of-flight mass spectrometry. Int J Mol Sci 13: 14518-14532, 2012.

4. Zhang Q, Wang J, Dong R, Yang S and Zheng S: Identification of novel serum biomarkers in child nephroblastoma using proteomics technology. Mol Biol Rep 38: 631-638, 2011.

5. Kahar UM, Chan KG, Salleh MM, Hii SM and Goh KM: A high molecular-mass Anoxybacillus sp. SK3-4 amylopullulanase: Characterization and its relationship in carbohydrate utilization. Int J Mol Sci 14: 11302-11318, 2013.

6. Wang J, Wang L, Zhang D, Fan Y, Jia Z, Qin P, Yu J, Zheng S and Yang F: Identification of potential serum biomarkers for Wilms tumor after excluding confounding effects of common systemic inflammatory factors. Mol Biol Rep 39: 5095-5104, 2012.

7. Al-Hussain T, Ali A and Akhtar M: Wilms tumor: An update. Adv Anat Pathol 21: 166-173, 2014.

8. Ehrlich PF: Bilateral Wilms' tumor: The need to improve outcomes. Expert Rev Anticancer Ther 9: 963-973, 2009.

9. Datta J and Vollmer CM Jr: Investigational biomarkers for pancreatic adenocarcinoma: Where do we stand? South Med J 107: 256-263, 2014.

10. Breslow NE, Palmer NF, Hill LR, Buring $J$ and d'Angio GJ: Wilms' tumor: Prognostic factors for patients without metastases at diagnosis. Results of the national Wilms' tumor study. Cancer 41: 1577-1589, 1978.
11. Yousuf FA and Iqbal MP: Review: Apolipoprotein E (Apo E) gene polymorphism and coronary heart disease in Asian populations. Pak J Pharm Sci 28: 1439-1444, 2015.

12. Gaudet D, Brisson D, Tremblay K, Alexander VJ, Singleton W, Hughes SG, Geary RS, Baker BF, Graham MJ, Crooke RM, et al: Targeting APOC3 in the familial chylomicronemia syndrome. N Engl J Med 371: 2200-2206, 2014.

13. Chan DC, Wong AT, Pang J, Barrett PH and Watts GF: Inter-relationships between proprotein convertase subtilisin/kexin type 9, apolipoprotein C-III and plasma apolipoprotein B-48 transport in obese subjects: A stable isotope study in the postprandial state. Clin Sci (Lond) 128: 379-385, 2015.

14. OoiEM,BarrettPH,Chan DC and Watts GF: ApolipoproteinC-III: Understanding an emerging cardiovascular risk factor. Clin Sci (Lond) 114: 611-624, 2008.

15. Geach T: Genetics. APOC3 mutations lower CVD risk. Nat Rev Cardiol 11: 496, 2014

16. Ji H, Ai N, Li Q, Zhang K and Di W: Clinical pathologies of breast cancer in the elderly and youths and their prognosis. Pak $\mathrm{J}$ Med Sci 30: 535-538, 2014. 